\title{
Workshop of Physical Agents 2009
}

\author{
Joaquín López \\ Editor of the special issue on Waf'08 \\ E-mail: Joaquin@uvigo.es
}

\begin{abstract}
The Workshop of Physical Agents intends to be a forum for information and experience exchange in different areas regarding the concept of agent on physical environments, especially applied to the control and coordination of autonomous systems: robots, mobile robots, industrial processes or complex systems. This special issue is devoted to the selected papers presented at the WAF'08 that took place from September 10th to 13th in the city of Vigo (SPAIN).
\end{abstract}

Index Terms-Waf, physical agents

\section{The Workshop of Physical Agents, WAF'2008}

$\mathbf{T}$ HIS issue of the Journal of Physical Agents (JoPhA) is devoted to the selected papers presented at the Workshop of Physical Agents (WAF) 2008 that took place from September 10th to 13th in the city of Vigo (SPAIN). For those that are not familiar with the workshop, allow me to explain a little bit about the WAF in general and the IX edition in particular.

The Workshop of Physical Agents intends to be a forum for information and experience exchange in different areas regarding the concept of agent on physical environments, especially applied to the control and coordination of autonomous systems: robots, mobile robots, industrial processes or complex systems. This meeting is a forum of discussion for International and Spanish Research Groups of associated areas related to the Autonomous Control Systems and the Artificial Intelligence applied to robotics. We had contributions describing scientific works in different areas that use agent-based technologies concepts, robotics, vision, (software agents) highlighting the similarities and synergies among physical and software agents. We understand that physical agent applications are mainly oriented to the control, coordination and planning of autonomous systems: industrial or mobile robots, industrial processes, domotics and complex systems.

The IX edition was once again a success. We started September 11th enjoying the plenary talk "Probabilistic Techniques for Robot Navigation" given by Professor Wolfram Burgard from the University of Freiburg, R.F.A). After that, during the rest of the day we had working sessions on Localization, Robots and Vision with some breaks to take a look at the posters and enjoy the different demos. Finally, on September 12th we enjoyed the plenary talk "Social Robots" given by Professor Miguel Angel Salichs from the University of Carlos III, Spain. After that, we had a working session on Applications, a working session on Agents and, after lunch we had more demos. Talks on the different working sessions can be seen form the UVigoTV web page (http://www.tv.uvigo.es/).

Joaquín López is with the department of Ingeniería de Sistemas y Automática, Universidad de Vigo. Campus de Lagoas-Marcosende 36200 Vigo, PO. SPAIN.
As a summary, we had sixteen papers presented on the different sessions and nine posters.

As in former editions of this workshop, the live demos and exhibitions brought significant media press such as TV and newspapers to the event. We had a chance to see an industrial robot brought by FANUC, challenging conference attendees to "work" faster than him. We could also interact with mobile social robots such as RATO from the University of Vigo and watch robots that play soccer from the University of Rey Juan Carlos and Alicante, surveillance robots, humanoids, etc. In this publication we include six papers about different topics of SLAM, agents and applications. Even though Localization and SLAM has extensively been studied and some solutions have been presented, it is still a challenging research topic and we included the first three papers of this issue to further analyze it.

The first one "MSISpIC: A Probabilistic Scan Matching Algorithm Using a Mechanical Scanned Imaging Sonar" compares two well known scan matching algorithms: the MbICP and the pIC and propose the MSISpIC, a probabilistic scan matching algorithm for the localization of an Autonomous Underwater Vehicle (AUV). The second one "SLAM and Map Merging" presents a multi-robot mapping and localization system. The approach uses a team of mobile robots which use scan-matching and fast-slam techniques for mapping. The third paper on this topic "Localization through omnivision for a tour-guide robot" presents an algorithm that solves the global localization based on a merit function that ranks different possible poses obtained from an acquired image. It uses an iterative process for the minimization of that function using a particle filter.

The next two papers are focused on applications. The first one "Pedestrian tracking using inertial sensors" proposes a personal navigation system based on the use of inertial sensors. The system is intended to be a compliment for the Global Positioning System in places where GPS signals are not available. The second one "Developing a Low-Cost Autonomous Indoor Blimp" describes the design of an autonomous blimpbased robot and its navigation system. The robot is based on a commercial kit and its dimensions were suitable for use in indoor environments.

The last paper of this issue "A Cognitive Approach to Multimodal Attention" presents a novel cognitive architecture which enables integrated and efficient filtering of multiple modality sensory information. The proposed attention mechanism is based on contexts that determine what data is relevant to the current situation.

And finally, I would like to thank all the people that collaborated with the journal and I would like to ask for 
your input regarding different aspects to improve it further. welcome.

Any thoughts, recommendations, or ideas will be more than 